
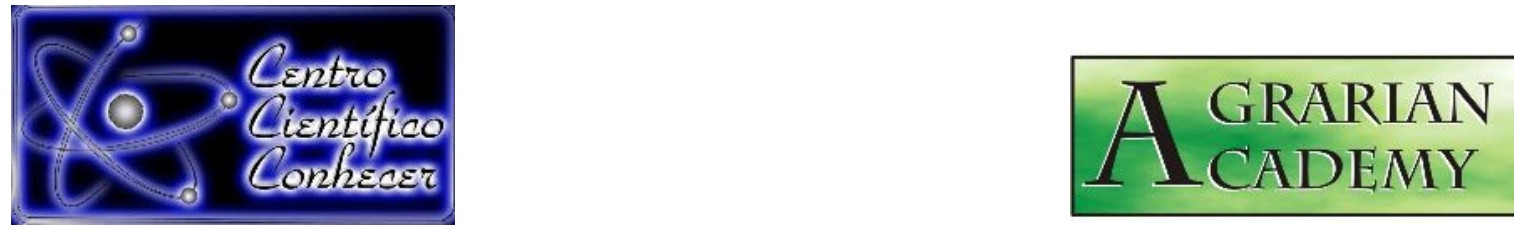

\title{
MINIMIZAÇÃO DE CUSTO NA FORMULAÇÃO DA RAÇÃO PARA BOVINOS DE CORTE USANDO FERRAMENTA DE OTIMIZAÇÃO
}

Rafaela Silva Cesca ${ }^{1}$ Rodrigo Couto Santos ${ }^{2}$ Ana Paula Cassaro Favarim ${ }^{3}$ Gismery da Silva Monteiro ${ }^{3}$ Karina Freitas Costa $^{4}$

1. Engenheira Física, Mestranda em Engenharia Agrícola, Faculdade de Ciências Agrárias-FCA, UFGD, Dourados-MS, Brasil (rafaela.s.cesca@gmail.com). DouradosBrasil;

2. Engenheiro Agrícola, Professor Doutor, Faculdade de Ciências Agrárias-FCA, UFGD;

3. Engenheira Civil, Mestranda em Engenharia Agrícola da Faculdade de Ciências Agrárias-FCA, UFGD;

4. Bióloga, UNIGRAN, Dourados - MS;

Recebido em: 02/06/2019 - Aprovado em: 15/06/2019 - Publicado em: 22/07/2019 DOI: 10.18677/Agrarian_Academy_2019a21

\begin{abstract}
RESUMO
A demanda global por produtos pecuários tem aumentado em consequência do aumento da população mundial. Na produção bovina é comum estudar a quantidade e qualidade das pastagens e se tratando de países de clima tropical, a disponibilidade das forrageiras está diretamente relacionada às condições climáticas locais. Devido a tais fatos, uma das saídas empregadas é a utilização de alimentação suplementar, com os concentrados comerciais, silagem ou feno e grãos, que muitas vezes elevam o custo do produto final. Diante do exposto este estudo teve como objetivo usar o método de programação linear por meio de otimização para formular uma ração com custo mínimo para gado de corte. Utilizou-se nessa modelagem um banco de dados das composições químico-bromatológicas de alimentos, que possibilitassem um ganho diário de peso de 1,2 kg para bovinos entre $250 \mathrm{~kg}$ e 453,6 kg. Foi gerado um algoritmo de otimização global determinístico com restrição nas variáveis de entrada baseado na técnica de cobertura de espaço não uniforme. Para a construção do modelo utilizou-se o software específico e as variáveis milho, cana de açúcar, forragem e suplemento. Após a construção do modelo verificou-se a quantidade otimizada de cada um desses elementos de forma a se ter o menor custo de ração no final do processo. Com o uso da otimização, foi possível atingir o menor custo na formulação de ração atendendo aos requisitos nutricionais tornando essa ferramenta importante na gestão sustentável da produção pecuária.
\end{abstract}

PALAVRAS-CHAVE: Alimentação suplementar, pecuária, simulação 


\title{
MINIMIZING COST IN FORMATION OF RATION FOR CUTTING CATTLE USING OPTIMIZATION TOOL
}

\begin{abstract}
Global demand for livestock products has increased as a result of rising world population. In cattle production, it is common to study the quantity and quality of pastures and, in tropical countries, the availability of forages is directly related to local climatic conditions. Due to such facts, one of the outputs employed is the use of supplementary feed, with commercial concentrates, silage or hay and grains, which often raise the cost of the final product. In view of the above, this study had as objective to use the linear programming method by means of optimization to formulate a ration with minimum cost for beef cattle. A database of chemical and bromatological compositions of foods was used in this model, which allowed a daily gain of $1.2 \mathrm{~kg}$ for cattle between $250 \mathrm{~kg}$ and $453.6 \mathrm{~kg}$. A deterministic global optimization algorithm with restriction on input variables was generated based on nonuniform space coverage technique. For the construction of the model was used the specific software and the variables corn, sugar cane, forage and supplement. After the construction of the model, it was verified the optimized amount of each of these elements in order to have the lowest feed cost at the end of the process. With the use of optimization, it was possible to achieve the lowest cost in the formulation of ration meeting the nutritional requirements making this tool important in the sustainable management of livestock production.
\end{abstract}

KEYWORDS: Supplementary feeding, cattle raising, simulation.

\section{INTRODUÇÃO}

Segundo Rojas-Downing et al. (2017) a demanda global por produtos pecuários deverá dobrar até 2050 , consequência do aumento da população mundial. Neste período, as mudanças climáticas serão ameaças constantes à produção bovina, devido ao impacto sobre a qualidade da forragem, disponibilidade de água, influencia na reprodução e estresse.

Ao abordar o tema "alimentação animal" para a produção bovina é comum estudar a quantidade e qualidade das pastagens envolvidas no processo. Entretanto, se tratando de países de clima tropical, a disponibilidade das forrageiras está diretamente relacionada às condições climáticas locais (BUTTLER et al., 2019). Conforme Ergon et al. (2018), em determinadas épocas do ano, as estiagens ocasionadas no inverno ou outono reduzem significativamente a quantidade e a qualidade da forragem, ao ponto de comprometer a produção de carne ou leite.

Os impactos na quantidade e qualidade de forragem dependem da região e do período de crescimento sendo que variações climáticas ocasionadas pelas épocas do ano podem até mesmo tornar uma área improdutiva (BERNABUCCI 2019). Um aumento de $2{ }^{\circ} \mathrm{C}$ produz impactos negativos sobre a produção de pasto e pecuária ocasionando a necessidade de suplementação alimentar com ração (SOUSA JUNIOR et al., 2019).

Segundo Castillo et al., (2019) a ciência tem mostrado que a ingestão de carne e produtos lácteos é benéfica para a nutrição humana dada suas propriedades nutricionais, porém tem se tornado cada vez mais necessário o desenvolvimento de um sistema pecuário intensivo que seja sustentável com o meio ambiente. Este remete a uma alimentação dos animais com produtos naturais e comercialmente mais econômicos.

Devido a tais fatos, uma das saídas empregadas no campo é a utilização de 
alimentação suplementar, com os concentrados comerciais, silagem ou feno e grãos, que muitas vezes elevam o custo do produto final (IDEHARA; RODRIGUES, 2011). Salami et al., (2019) afirmam que a formulação de rações é um problema recorrente para os criadores bovinos. Geralmente, a ração é feita especificando-se os requisitos nutricionais entre outros parâmetros, sendo que o uso de um algoritmo é a melhor forma de encontrar uma formulação viável e econômica.

Diante do exposto este estudo teve o objetivo de usar o método de programação linear - otimização e formular uma ração de custo mínimo para gado de corte com peso médio de $250 \mathrm{~kg}$, de exigência nutricional diária para ganho de peso de $1,2 \mathrm{~kg} / \mathrm{dia}$.

\section{MATERIAL E MÉTODOS}

A pesquisa foi realizada na Faculdade de Ciências Agrárias (FCA) da Universidade Federal da Grande Dourados (UFGD), localizado no município de

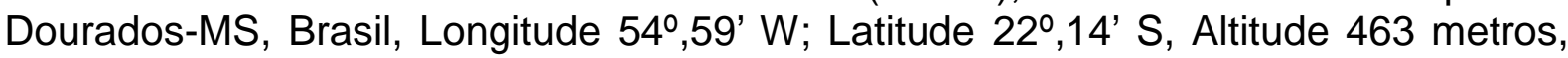
com clima segundo Koppen classificado como Am Monçonico, com inverno seco, precipitação média anual de $1500 \mathrm{~mm}$ e temperatura média de $22^{\circ} \mathrm{C}$ (ALVARES et al., 2013).

O banco de dados das composições químico-bromatológicas dos alimentos utilizados neste estudo foi gerado por Rodrigues (2009) e em informações do NRC (1996) para um ganho diário esperado de peso de 1,2 kg para bovinos entre $250 \mathrm{~kg}$ e 453,6 kg, de acordo com a previsão ideal, conforme Tabelas 1 e Tabela 2. Os valores considerados para os produtos foram referenciados por Clicmercado (2019) e MFrural (2019) mês base abril / 2019, conforme Tabela 3.

TABELA 1. Previsão de dados de NRC (1996)

\begin{tabular}{|l|c|c|c|l|l|l|}
\hline PESO (kg) & $\begin{array}{c}\text { MS } \\
\text { (kg/dia) }\end{array}$ & $\begin{array}{c}\text { NDT } \\
\text { (kg/dia) }\end{array}$ & $\begin{array}{c}\text { EM } \\
\text { (Mcal/kg) }\end{array}$ & PB (\% MS) & Ca (\% MS) & P (\% MS) \\
\hline 250.00 & 5.00 & 4.5 & 2.74 & 12.40 & 0.49 & 0.24 \\
\hline
\end{tabular}

MS = matéria seca; NDT = nutrientes diários totais; $\mathrm{EM}=$ energia metabolizável; PB = proteína bruta; $\mathrm{Ca}=$ cálcio; $\mathrm{P}=$ Fósforo

TABELA 2. Avaliação químico-bromatológica de alimentos indicados por NRC (1996) e Rodrigues (2009)

\begin{tabular}{|l|r|r|r|r|}
\hline \multicolumn{1}{|c|}{ PRODUTO } & \multicolumn{1}{c|}{$\begin{array}{c}\text { MILHO } \\
\text { (FARELO) }\end{array}$} & $\begin{array}{c}\text { CANA DE } \\
\text { AÇUCAR } \\
\text { (BAGAÇO) }\end{array}$ & \multicolumn{1}{c|}{$\begin{array}{c}\text { ALFAFA } \\
\text { (FENO) }\end{array}$} & SUPLEMENTO \\
\hline Matéria Seca (\%) & 22.85 & 91.95 & 85.00 & 0 \\
\hline NDT (\% MS) & 60.84 & 46.64 & 67.63 & 0 \\
\hline EM (Mcal/kg) & 2.20 & 1.69 & 2.45 & 0 \\
\hline PB (\% MS) & 9.58 & 1.36 & 22.39 & 26.3 \\
\hline Ca (g/kg) & 0.27 & 0.00 & 1.14 & 7.5 \\
\hline P (g/kg) & 0.20 & 0.00 & 0.45 & 0.15 \\
\hline CUSTO (US\$/kg) & 0.16 & 0.13 & 1.35 & \\
\hline
\end{tabular}

MS = matéria seca; NDT = nutrientes diários totais; $\mathrm{EM}=$ energia metabolizável; $\mathrm{PB}$ = proteína bruta; $\mathrm{Ca}=$ cálcio; $\mathrm{P}$ = Fósforo 
TABELA 3. Valores atuais dos alimentos. Fonte: Clicmercado (2019) e MFrural (2019), adaptado

\begin{tabular}{|c|c|c|}
\hline ALIMENTO & $\begin{array}{c}\text { Preço comercial (R\$/ } \\
\text { unidade) }\end{array}$ & Preço unitário (R\$/Kg) \\
\hline MILHO (FARELO) & $38,50 / 60$ & 0,64 \\
\hline $\begin{array}{c}\text { CANA DE AÇUCAR } \\
\text { (BAGAÇO) }\end{array}$ & $130,00 / 1000$ & 0,13 \\
\hline ALFAFA (FENO) & $1350,00 / 1000$ & 1,35 \\
\hline
\end{tabular}

Segundo Faddoul et al. (2018) e um algoritmo de otimização global determinístico para problemas com restrição nas variáveis de entrada baseia-se na técnica de cobertura de espaço não uniforme, sendo que para o estudo proposto a formulação matemática foi obtida seguindo a dedução:

Função Objetivo: Min $\mathrm{Q}(\mathrm{x})=$

$\sum_{j=1}^{n} C_{j}^{T} \cdot x_{j}$

Restrições:

$\sum_{j=1}^{n} a_{i j} \cdot x_{j} \geq b_{i}(\mathrm{i}=1,2,3, \ldots, \mathrm{m})$

e $x_{j} \geq 0 \quad(\mathrm{j}=1,2,3, \ldots, \mathrm{n})$

em que,

$\mathrm{c}_{\mathrm{j}}$ - lucro de cada unidade do produto j;

$\mathrm{x}_{\mathrm{j}}$ - quantidade do produto j utilizada;

$a_{i j}$ - quantidade do nutriente i contida por unidade do componente j;

$\mathrm{b}_{\mathrm{i}}$ - quantidade do nutriente i necessária.

Para a construção do modelo utilizou-se o software LINDO 6.1® sendo:

$! \mathrm{M}=$ Milho, $\mathrm{C}=$ Cana de Açúcar (bagaço), $\mathrm{A}=$ Alfafa (feno) e $\mathrm{S}=$ suplemento.

A programação ficou construída da forma:

!Minimizar o custo de ração

MIN $0.64 \mathrm{M}+0.13 \mathrm{C}+1.35 \mathrm{~A}+0.60 \mathrm{~S}$

ST

!restrição 1: necessidade de Matéria Seca diária

MS) $0.2285 \mathrm{M}+0.9195 \mathrm{C}+0.85 \mathrm{~A}>=5$

!restrição 2: necessidade de Nutrientes Digestivos Totais diário

NDT) $0.6084 \mathrm{M}+0.4664 \mathrm{C}+0.6763 \mathrm{~A}>=4.5$

!restrição 3: Energia Metabólica gasta na digestão diária

EM) $2.20 \mathrm{M}+1.69 \mathrm{C}+2.45 \mathrm{~A}>=2.74$

!restrição 4: necessidade de Proteína Bruta diária

PB) $0.0958 \mathrm{M}+0.136 \mathrm{C}+0.2239 \mathrm{~A}>=0.62$

!restrição 5: necessidade de Cálcio diário

Ca) $0.27 \mathrm{M}+1.14 \mathrm{~A}+26.3 \mathrm{~S}>=2.45$

!restrição 6: necessidade de Fósforo diário

P) $0.20 \mathrm{M}+0.45 \mathrm{~A}+7.5 \mathrm{~S}>=1.2$

! não negatividade

$M>=0$ 
END

Após a construção do modelo verificou-se a quantidade otimizada de Milho, Cana de Açúcar (bagaço), Alfafa (feno) e Suplemento de forma a ter o menor custo de ração no final do processo.

\section{RESULTADOS E DISCUSSÃO}

Com os dados de entrada propostos na metodologia, pelo software LINDO $6.1{ }^{\circledR}$ obteve-se os resultados da Tabela 4, 5 e 6 , dispostos na mesma interface do aplicativo, traduzidos para o idioma Português (Brasil): Na Tabela 4 observou-se que o resultado da função objetivo da formulação indicou um valor de 1.35. Ou seja, os dados gerados afirmam que é possível minimizar o custo da ração em aproximadamente $R \$ 1,35$. Estudo realizado por Belanche et al. (2019) demonstraram a eficiência da otimização como ferramenta de gestão na pecuária ao verificar a diminuição da influência da sazonalidade anual em termos de porcentagem de animais em ordenha e produção de leite, permitindo minimizar custos com manejo de animais considerados fora de temporada.

TABELA 4. Valor da função objetivo

\begin{tabular}{|c|c|c|}
\hline \multicolumn{3}{|c|}{ FUNÇÃO OBJETIVO: 1.350288} \\
\hline VARIÁVEL & VALOR & REDUÇÃO DE CUSTO \\
\hline M & 0.000000 & 0.454420 \\
\hline C & 9.648371 & 0.000000 \\
\hline A & 0.000000 & 1.125494 \\
\hline S & 0.160000 & 0.000000 \\
\hline
\end{tabular}

$\mathrm{M}=$ Milho; $\mathrm{C}=$ Cana de açúcar; $\mathrm{A}=$ Alfafa e $\mathrm{S}=$ Suplemento.

No modelo otimizado as variáveis utilizadas obtiveram valores de $M=$ Milho, $\mathrm{C}=$ Cana de Açúcar (bagaço), $\mathrm{A}=$ Alfafa (feno) e $\mathrm{S}=$ suplemento. iguais a $\mathrm{M}=0$; $\mathrm{C}=9.648371 ; \mathrm{A}=0$ e $\mathrm{S}=0.1600$ (Tabela 4). Este resultado indicou que a Cana de açúcar (bagaço) e Suplemento são básicas, justificando o investimento nas mesmas.

Ao analisar os nutrientes diários totais (NDT) e Fósforo (P) (Tabela 5) verificou-se que os mesmos apresentaram folga nula, não tendo ociosidade. Os valores de "preço sombra" foram de -0.278731 e -0.080000 . Já as outras restrições apresentaram excedente, ou seja, podem ser utilizadas conforme a quantidade formulada sem influenciar no custo final.

TABELA 5. Faixas laaterais sda direita

\begin{tabular}{|c|c|c|}
\hline LINHA & FOLGA OU EXCEDENTE & PREÇO SOMBRA \\
\hline MS & 3.871677 & 0.000000 \\
\hline NDT & 0.000000 & -0.278731 \\
\hline EM & 13.565746 & 0.000000 \\
\hline PB & 0.692178 & 0.000000 \\
\hline CA & 1.758000 & 0.000000 \\
\hline P & 0.000000 & -0.080000 \\
\hline
\end{tabular}

MS = matéria seca; NDT = nutrientes diários totais; $\mathrm{EM}=$ energia metabolizável; $\mathrm{PB}=$ proteína bruta; $\mathrm{Ca}=$ cálcio; $\mathrm{P}=$ Fósforo. 
Uyeh et al. (2018) também utilizaram um método simplificado de otimização na pecuária, aplicado a um sistema de controle de ração para engorda de bovinos de corte de forma a avaliar a utilização de nutrientes em diversas situações. O método foi estudado com a finalidade de reduzir os custos de alimentação e ao final da pesquisa ficou demonstrada a eficiência do modelo em suprir as exigências nutricionais de alimentação para bovinos de corte a baixo custo.

Ao observar a Tabela 6, a variável Cana de açúcar (C) permite um aumento de até 0.348359 , de modo que elevando o valor até esse patamar, está ainda assim será uma variável básica que supera as variáveis Milho $(M)$ e Alfafa (A) que apresentaram valor classificado como INFINITO. No que diz respeito ao decréscimo, esta pode ser reduzida até 0.130000 que ainda mantém inalteradas as variáveis Milho e Alfafa. A outra variável básica, Suplemento, permite um aumento de 17.040758 e decréscimo de 0.600000 , de forma a proporcionar valor igual a 0 (zero) às variáveis Milho e Alfafa. Conforme Uyeh et al. (2018) a otimização de processos utilizando a adoção de algoritmos na resolução de problemas de formulação de rações proporcionam dietas de menor custo que satisfazem as necessidades específicas de nutrientes.

TABELA 6. Faixas coeficientes objetivas

\begin{tabular}{|c|c|c|c|}
\hline VARIÁVEL & COEF ATUAL & $\begin{array}{c}\text { AUMENTO } \\
\text { ADMISSÍVEL }\end{array}$ & $\begin{array}{c}\text { DIMINUIČÃO } \\
\text { ADMISSIVEL }\end{array}$ \\
\hline $\mathbf{M}$ & 0.640000 & INFINITO & 0.454420 \\
\hline $\mathbf{C}$ & 0.130000 & 0.348359 & 0.130000 \\
\hline $\mathbf{A}$ & 1.350000 & INFINITO & 1.125494 \\
\hline S & 0.600000 & 17.040758 & 0.600000 \\
\hline
\end{tabular}

M= Milho; $C=$ Cana de açúcar; $A=$ Alfafa e $S=$ Suplemento.

Segundo Zhao e Guan (2018) o uso de ferramenta de otimização é importante ao tratar com modelos determinísticos, como os deste estudo, pois a distribuição real dos parâmetros aleatórios é geralmente desconhecida e difícil de prever com precisão e a estimativa imprecisa da distribuição verdadeira pode levar a soluções próximas ao ideal.

Vale ressaltar que o baixo custo nem sempre é o objetivo principal procurado na formulação adequada de uma ração. Segundo Siqueira et al. (2017) as mudanças climáticas tem causado secas frequentes e prolongadas, com perdas de pastos para ruminantes criados à solta ou suplementos volumosos produzidos com baixa qualidade. Já em Bulgogi, uma cozinha popular coreana, por exemplo, é exigida que a carne consumida tenha um atributo sensorial específico (em termos de sabor e maciez) que geralmente são obtidos pelo tipo de alimento fornecido ao animal, neste caso; grandes quantidades de concentrados na ração (PARK et al., 2012).

\section{CONCLUSÕES}

Para a formulação de ração para bovinos de corte, o conjunto do bagaço de cana de açúcar e suplemento foi o que proporcionou maior minimização do custo da ração.

Com o uso da otimização foi possível atingir o menor custo na formulação de ração atendendo aos requisitos nutricionais tornando essa ferramenta importante na gestão sustentável da produção pecuária.

O trabalho focado na formulação nutricional de bovinos pelo processo de modelagem matemática e programação linear, apresentou resultado confiável AGRARIAN ACADEMY, Centro Científico Conhecer - Goiânia, v.6, n.11; p. 224 
envolvendo fatores determinísticos e conhecimento sobre alimentação de ruminantes.

\section{REFERÊNCIAS}

ALVARES, C.A.; STAPE, J.L.; SENTELHAS, P.C.; MORAES, G.L.; SPARAVEK, G. Köppen's climate classification map for Brazil. Meteorologische Zeitschrift. v.22, n.6, p.711-728, 2013. Disponível em: https://doi.org/10.1127/0941-2948/2013/0507 Doi: 10.1127/0941-2948/2013/0507

BELANCHE A., MARTÍN-GARCÍA A.I., FERNÁNDEZ-ÁLVAREZ J., PLEGUEZUELOS J., MANTECÓN A.R., YÁÑEZ-RUIZ D.R. Optimizing management of dairy goat farms through individual animal data interpretation: A case study of smart farming in Spain, Agricultural Systems. v.173, p.27-38, 2019. Disponível em: https://doi.org/10.1016/j.agsy.2019.02.002 Doi: 10.1016/j.agsy.2019.02.002

BERNABUCCI, U. Climate change: impact on livestock and how can we adapt. Animal Frontiers, v. 9, p. 3-5, 2019, Disponível em: https://doi.org/10.1093/af/vfy039 Doi: https://doi.org/10.1093/af/vfy039

BUTTLER, A.; MARIOTTE, P.; MEISSER, M.; GUILLAUME, T.; SIGNARBIEUX, C.; VITRA, A.; et al.,; Drought-induced decline of productivity in the dominant grassland species Lolium perenne L. depends on soil type and prevailing climatic conditions, Soil Biology and Biochemistry, v.132, p. 47-57, 2019, Disponível em: https://doi.org/10.1016/j.soilbio.2019.01.026 Doi: 10.1016/j.soilbio.2019.01.026.

CASTILlO, C.; ABUElO, A.; HERNÁNDEZ, J. Ruminant (Bovine, Caprine, and Ovine) Milk and Meat Production: The Challenge of Food Quality and Sustainability Through the Use of Plant Extracts, Encyclopedia of Food Security and Sustainability, Elsevier, p. 25-42, 2019, Disponível em: https://doi.org/10.1016/B978-0-08-100596-5.22187-2 Doi: 10.1016/B978-0-08100596-5.22187-2.

CLICMERCADO. MERCADO: Corretora de Mercadorias. Agrobussines brasileiro Cotações. Representações CGF Ltda. 2019.

ERGON, Å.; SEDDAIU, G.; KORHONEN, P.; VIRKAJÄRVI, P.; BELLOCCHI, G.; JØRGENSEN, M.; ØSTREM, L.; REHEUL, D.; VOLAIRE, F. How can forage production in Nordic and Mediterranean Europe adapt to the challenges and opportunities arising from climate change?. European Journal of Agronomy, v. 92, p. 97-106, 2018, Disponível em: https://doi.org/10.1016/j.eja.2017.09.016 Doi: 10.1016/j.eja.2017.09.016.

FADDOUL, R.; RAPHAEL, W.; CHATEAUNEUF, A. Maintenance optimization of series systems subject to reliability constraints, Reliability Engineering \& System Safety, $\quad$ v. 180, p. 179-188, 2018, Disponível em: https://doi.org/10.1016/j.ress.2018.07.016 Doi: 10.1016/j.ress.2018.07.016.

IDEHARA, S.J; RODRIGUES, R.C. Otimização da Alimentação Bovina através de Programação Linear. Embrapa Clima Temperado Pelotas, RS. 2011. 
MFRURAL: $O$ agronegócio passa aqui. Anúncios de Produtos Rurais e Agropecuários. Representações CGF Ltda. 2019.

NRC - National Research Council. Nutrients requeriments of beef cattle. Washington, D.C. v.7, p.244, 1996

PARK, S.H.; HAM, S.; LEE, M.A. How to improve the promotion of Korean beef barbecue, bulgogi, for international customers. An application of quality function deployment. Appetite. v.59, n.2, p.324-332, 2012. Disponível em: https://doi.org/10.1016/j.appet.2012.05.008 Doi: 10.1016/j.appet.2012.05.008

RODRIGUES, R. C. Avaliação químico-bromatológica de alimentos produzidos em Terras Baixas para nutrição animal. Pelotas: Embrapa Clima Temperado, v.270, p.28, 2009

ROJAS-DOWNING M.M., NEJADHASHEMI A.P., HARRIGAN T., WOZNICKI S.A. Climate change and livestock: Impacts, adaptation, and mitigation. Climate Risk Management. v.16, p.145-163, 2017. Disponível em https://doi.org/10.1016/j.crm.2017.02.001 Doi: 10.1016/j.crm.2017.02.001

SALAMI, S.A.; LUCIANO, G.; O'GRADY, M.N.; BIONDI, L.; NEWBOLD, C.J.; KERRY, J.P.; PRIOLO, A. Sustainability of feeding plant by-products: A review of the implications for ruminant meat production, Animal Feed Science and Technology, v. 251, p. 37-55, 2019, Disponível em: https://doi.org/10.1016/j.anifeedsci.2019.02.006 Doi: 10.1016/j.anifeedsci.2019.02.006.

SIQUEIRA, M.C.B.; FERREIRA, M.A.; MONNERAT, J.P.I.S.; SILVA, J.L.; COSTA, C.T.F.; CONCEIÇÃO, M.G. et al. Optimizing the use of spineless cactus in the diets of cattle: Total and partial digestibility, fiber dynamics and ruminal parameters, Animal Feed Science and Technology, v. 226, p. 56-64, 2017, Disponível em: https://doi.org/10.1016/j.anifeedsci.2016.12.006 10.1016/j.anifeedsci.2016.12.006.

SOUSA JUNIOR, W.T., MONTEVECHI, J.A.B., MIRANDA, R.C., CAMPOS, A.T. Discrete simulation-based optimization methods for industrial engineering problems: A systematic literature review, Computers \& Industrial Engineering, v.128, p.526540, 2019. Disponível em: https://doi.org/10.1016/j.cie.2018.12.073 Doi: 10.1016/j.cie.2018.12.073

UYEH D.D., MALLIPEDDI R., PAMULAPATI T., PARK T., KIM J., WOO S., HA Y. Interactive livestock feed ration optimization using evolutionary algorithms. Computers and Electronics in Agriculture. v.155, p.1-11, 2018. Disponível em: https://doi.org/10.1016/j.compag.2018.08.031. Doi: 10.1016/j.compag.2018.08.031.

ZHAO C; GUAN Y. Data-driven risk-averse stochastic optimization with Wasserstein metric, Operations Research Letters, v. 46, n. 2, p. 262-267, 2018, Disponível em https://doi.org/10.1016/j.orl.2018.01.011. Doi: 10.1016/j.orl.2018.01.011. 